Registration accuracy in multilevel soft lithography

This content has been downloaded from IOPscience. Please scroll down to see the full text. 2007 Nanotechnology 18175302

(http://iopscience.iop.org/0957-4484/18/17/175302)

View the table of contents for this issue, or go to the journal homepage for more

Download details:

IP Address: 130.237.165.40

This content was downloaded on 08/09/2015 at $21: 42$

Please note that terms and conditions apply. 


\title{
Registration accuracy in multilevel soft lithography
}

\author{
Stefano Pagliara, Luana Persano, Andrea Camposeo, \\ Roberto Cingolani and Dario Pisignano
}

National Nanotechnology Laboratory of INFM-CNR and Istituto Italiano di Tecnologia (IIT), c/o Distretto Tecnologico ISUFI, Università del Salento, via Arnesano, I-73100, Lecce, Italy

E-mail: stefano.pagliara@unile.it

Received 16 January 2007, in final form 5 March 2007

Published 2 April 2007

Online at stacks.iop.org/Nano/18/175302

\begin{abstract}
We investigate the registration accuracy achievable by multilevel soft lithography. By a specifically designed soft lithography aligner, we obtain, for the average misalignment between two registered patterned organic layers, values decreasing from $(4.96 \pm 0.02)$ to $(0.50 \pm 0.01) \mu \mathrm{m}$ upon increasing the Young's modulus of the stamp materials from 1.8 to $2600 \mathrm{MPa}$. This clearly identifies in the stamp distortions the main factor limiting the registration accuracy. The potentiality to achieve registration within $500 \mathrm{~nm}$ over areas of $50 \times 50 \mu \mathrm{m}^{2}$ is demonstrated, opening the way for soft lithographies with high overlay alignment accuracy.
\end{abstract}

(Some figures in this article are in colour only in the electronic version)

\section{Introduction}

Soft [1] and nanoimprint [2] lithographies have attracted a lot of attention because of their versatility. Nanoimprint lithography [2] transfers a pattern from a hard mould into a deformable material deposited onto a rigid substrate. This technique can suitably replicate structures as small as $5 \mathrm{~nm}$ [3] and it has been used to pattern Fresnel lenses [4] and metaloxide-semiconductor field-effect transistors [5]. The strong development of dedicated tools and resist materials over the last decade has further enhanced the potentiality of imprint lithographies, which have been included in the International Technology Roadmap for Semiconductors [6] as one of the strategic approaches for the $32 \mathrm{~nm}$ node. However, there are a few critical issues that must be addressed, such as the planarity constraint of the process, the need of anti-sticking layers for easy stamp-resist detachment [7] and the improvement of the polymer transport [8].

Soft lithography $[1,9,10]$ is even less expensive than nanoimprinting. Despite this, it allows one to pattern nonplanar surfaces [11] and it is applicable to large area patterning by single-step procedures and to a wide class of materials with many applications in photonics [12], electronics [13, 14], biotechnology and biology [15]. First proposed for patterning self-assembled monolayers of alkanethiols on gold by elastomeric stamps [10], soft lithography overcomes many of the shortcomings of conventional lithographies, enabling the control of the molecular structure and functionality of surfaces [16], and the pattern and manipulation of proteins and cells [17]. Molecular-size resolution has been demonstrated by soft lithographies both in-plane [9] and along the vertical dimension [18]. Recently, both elastomeric [19] and thermoplastic [20] elements have been proposed for soft imprinting processes, such as UV-moulding with [21] or without [19] externally applied pressures. In addition, microcontact printing has been applied to patterning proteins in complex geometries and microarrays and, since the molecule transfer is unidirectional from the elastomeric elements to the target surfaces, for printing multiple antigens on the same substrate [22]. Multiple soft lithography processes have also been applied to the realization of three-dimensional nanostructures [11] and microfluidic valves and pumps [23]. These results have raised the interest in multilayer soft lithography and in particular to the assessment of the best accuracy achievable in multilevel alignment by polymeric replicas. As opposed to multilevel optical lithography, which is very well established for microfabrication [24-26] and nanoimprinting [27, 28], the registration accuracy of multilevel soft lithography (M-SL) is still unexplored. $\mathrm{Hu}$ et al reported a registration within $12 \mu \mathrm{m}$ over $40 \mathrm{~mm}^{2}$ area in the fabrication of GaAs/AlGaAs field effect transistors using poly(dimethylsiloxane) (PDMS) moulds [14]. Recently, a freespace moiré fringe-based method has been proposed for the 


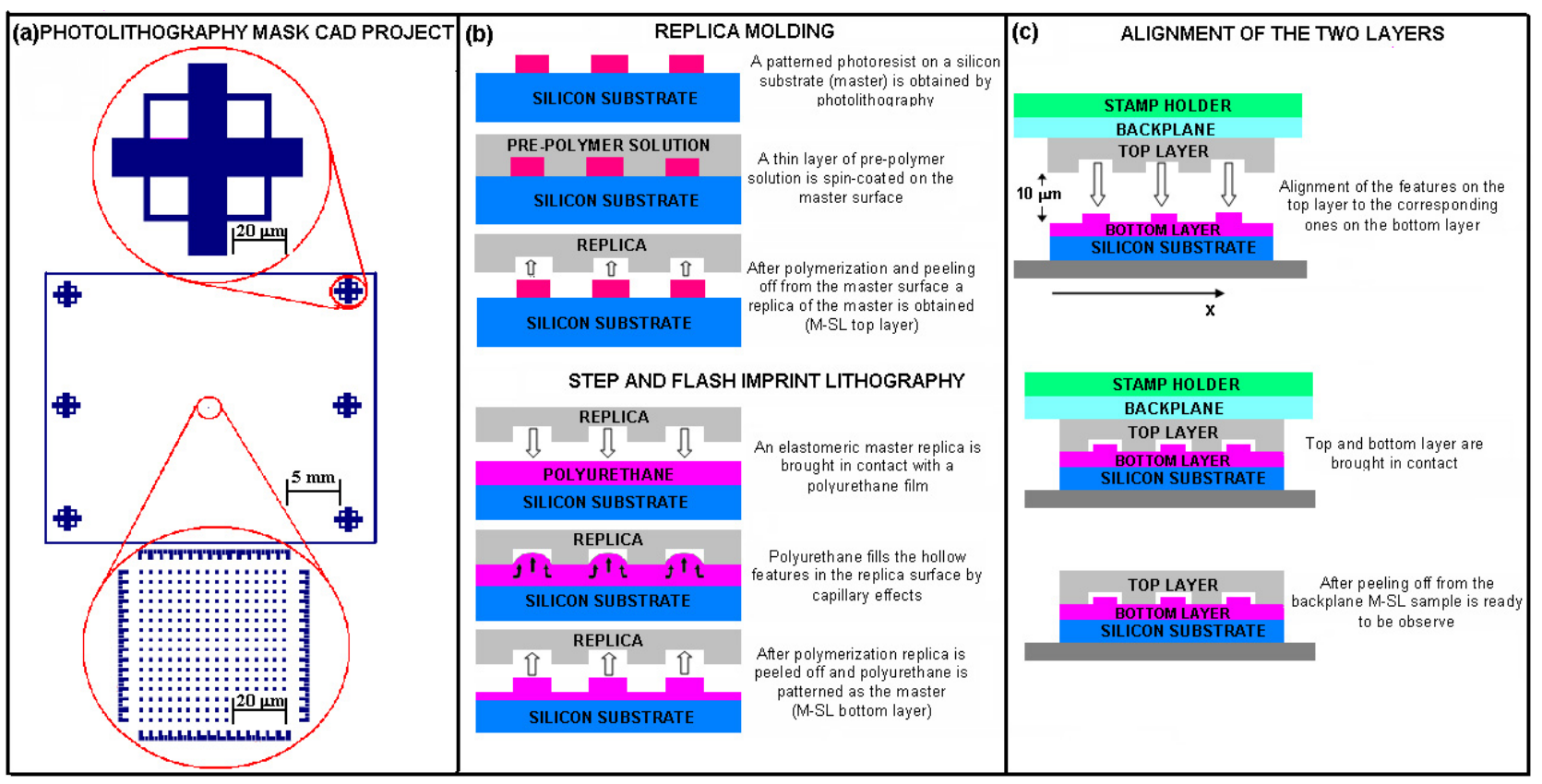

Figure 1. Technological steps of M-SL. (a) CAD design of Cr/quartz mask: $16 \times 16$ matrix of pitches ( $1 \mu \mathrm{m}$ side and $2 \mu \mathrm{m}$ distance), rulers $2 \mu \mathrm{m}$ thick and reference markers. (b) Realization of the M-SL elastomeric or thermoplastic top layer through replica moulding (top) and of the polyurethane M-SL bottom layer by SFIL (bottom). (c) Scheme of the alignment procedure. Features not to scale. Gaps in (c) schematize the insertion of the pitches of the bottom M-SL layer within the hollow features of the top M-SL layer.

alignment of optically transparent elements for layer-by-layer fabrication in soft lithography [29]. Since a main reason for the misalignment in M-SL is the occurrence of elastic deformations of the structures on the elastomeric stamps, ranging between hundreds of nanometres and about $1 \mu \mathrm{m}$ for PDMS areas of the order of $1 \mathrm{~cm}^{2}$ [30], different materials have to be tested as moulds in order to improve the registration accuracy.

In this work we present the first comprehensive study of the registration accuracy achievable in M-SL methods, as related to the elastic modulus of the polymer employed for the stamp. The multilevel alignment was accomplished using suitably designed, state-of-the-art alignment equipment and a pattern including 256 polymeric features with $1 \mu \mathrm{m}$ side, and tested on elastomeric and thermoplastic replicas. Alignment accuracies from about 5.0 to $0.5 \mu \mathrm{m}$ were obtained over an area of $50 \times 50 \mu \mathrm{m}^{2}$ by increasing the hardness of the stamp, and the potentiality to achieve registration within the range of tens of nanometres is demonstrated.

\section{Experimental details}

\subsection{Fabrication}

We fabricated the features on the top layer and on the bottom layer involved in the M-SL starting from a photoresist pattern generated by photolithography on a Si substrate. Figure 1(a) shows the design of the photolithographic mask, realized by electron-beam lithography by a Raith 150 system, and consisting of a $16 \times 16$ matrix of $1 \mu \mathrm{m}$ side pitches separated by $2 \mu \mathrm{m}$ and by four rulers $2 \mu \mathrm{m}$ thick. The pattern is surrounded by three pairs of reference markers (crosses) placed at distances of about $3.5 \mathrm{~cm}$ along the sides of the mask. Figure 1(b) summarizes the steps carried out to transfer the pattern from the photoresist to an elastomeric or thermoplastic stamp (top M-SL layer) and from an elastomeric stamp to a polyurethane film (bottom M-SL layer). For the top M-SL layer we fabricated $500 \mu \mathrm{m}$ thick stamps by replica moulding from the photoresist pattern, employing elastomeric materials (PDMS and the vinyl and hydrosilane polymer, hard-PDMS-h-PDMS) [31] and a thermoplastic olefin polymer of amorphous structure $\left(\right.$ TOPAS $\left.^{\circledR}\right)$ [20]. The Sylgard 184 PDMS base (A) and curing agent (B) are mixed in a ratio of nine parts $\mathrm{A}$ and one part $\mathrm{B}$, in weight, and the elastomer was spin-coated $(500 \mathrm{rpm}$ for $30 \mathrm{~s})$ on the master and polymerized in situ at $140{ }^{\circ} \mathrm{C}$ for $15 \mathrm{~min}$. The hPDMS solution was prepared mixing $3.4 \mathrm{~g}$ of trimethylsiloxyterminated vinylmethylsiloxane-dimethylsiloxane (VDT-731, ABCR, Karlsruhe, Germany), $18 \mu \mathrm{l}$ of a Pt catalyst (platinum divinyltetramethyldisiloxane, SIP6831.1, ABCR, Karlsruhe, Germany) and $5 \mu \mathrm{l}$ of a modulator $(2,4,6$, 8-tetramethyltetravinylcyclotetrasiloxane, Sigma Aldrich, St Louis, MO). After having degassed the mixture for 1$2 \mathrm{~min}, 1 \mathrm{~g}$ of methylhydrosiloxane-dimethylsiloxane (HMS301, ABCR, Karlsruhe, Germany) was added, and the h-PDMS solution was gently stirred. We then spin-coated (3000 rpm for $30 \mathrm{~s}$ ) a thin h-PDMS layer onto the master surface, and cured it at $60^{\circ} \mathrm{C}$ for $30 \mathrm{~min}$. After polymerization, Sylgard $184 \mathrm{PDMS}$, in the form of liquid pre-polymer, was spin-coated (500 rpm for $30 \mathrm{~s}$ ) onto the h-PDMS layer and cured at $60^{\circ} \mathrm{C}$ for $1 \mathrm{~h}$. Then, the composite stamp was carefully peeled off from the master surface. TOPAS ${ }^{\circledR}$ (grade 8007) pellets were dissolved in toluene (in a ratio of one part to three in weight) by sonification at $75^{\circ} \mathrm{C}$ for $2 \mathrm{~h}$. The solution was then spin- 

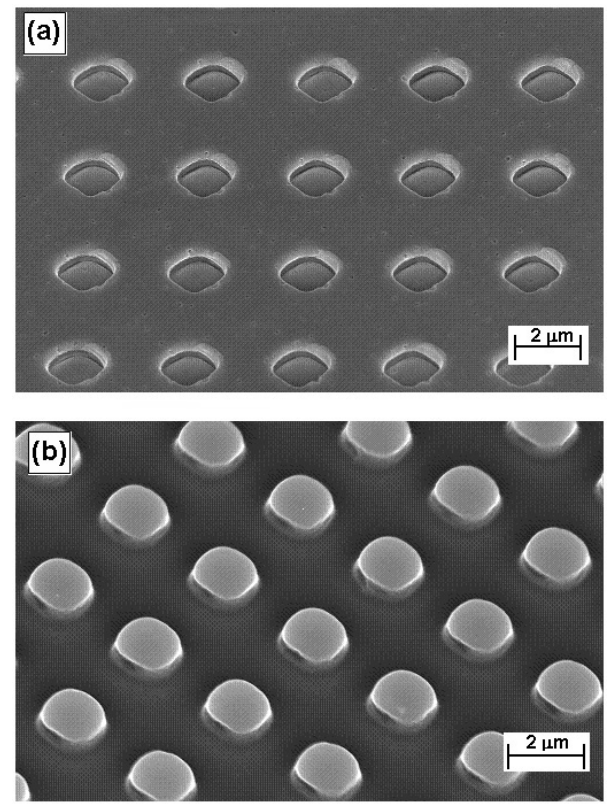

Figure 2. SEM images of the top (a) and of the bottom (b) M-SL patterns.

coated (3000 rpm for $40 \mathrm{~s}$ ) onto the master surface, and heated at $60^{\circ} \mathrm{C}$ for $2 \mathrm{~h}$ to let toluene evaporate. Afterwards, Sylgard 184 PDMS, in the form of liquid pre-polymer, was spin-coated (500 rpm for $30 \mathrm{~s}$ ) onto the TOPAS ${ }^{\circledR}$ layer and cured at $60^{\circ} \mathrm{C}$ for $1 \mathrm{~h}$. Finally, the composite stamp was carefully peeled off from the master surface.

For the bottom M-SL structure, we performed step and flash imprint lithography (SFIL) [19] to realize a negative copy of the top layer pattern on a thin layer (thickness $<1 \mu \mathrm{m}$ ) of Norland Optical Adhesive (NOA72 [32]) polyurethane, previously deposited on a $\mathrm{Si}$ substrate. In this soft lithographic procedure (figure 1(b)), the stamp is placed on the substrate, without preliminary surface treatment, to let the NOA72 fill the grooves of the mould by capillary force. Immediately after positioning the replica (i.e. during the capillary penetration) the system is exposed to UV light $(500 \mathrm{~W}$, wavelength $\cong$ $290 \mathrm{~nm}$ ) through the backside of the template, thereby crosslinking NOA72 at room temperature for $15 \mathrm{~min}$. After the polymerization of the polyurethane film, the mould is separated from the substrate, and the pattern remains on the target. The resulting features on the polyurethane film (M-SL bottom layer) are square-like pitches of $0.99-1.00 \mu \mathrm{m}$ side and height in the range $0.35-0.40 \mu \mathrm{m}$, whereas the corresponding hollow features in the stamp surface (M-SL top layer) are squarelike holes of $1.00-1.01 \mu \mathrm{m}$ side and $0.50-0.55 \mu \mathrm{m}$ depth (depending on the stamp material), as imaged by scanning electron microscopy (SEM). Since the lateral size of the polyurethane features on the bottom M-SL layer is slightly smaller than that of the hollow dots in the top layer, a precise alignment procedure between the two films can result in the insertion of each dot of the bottom layer matrix into the corresponding dot of the top layer matrix, as schematized in figure 1(c). Figure 2 shows SEM images of the top and bottom layers.

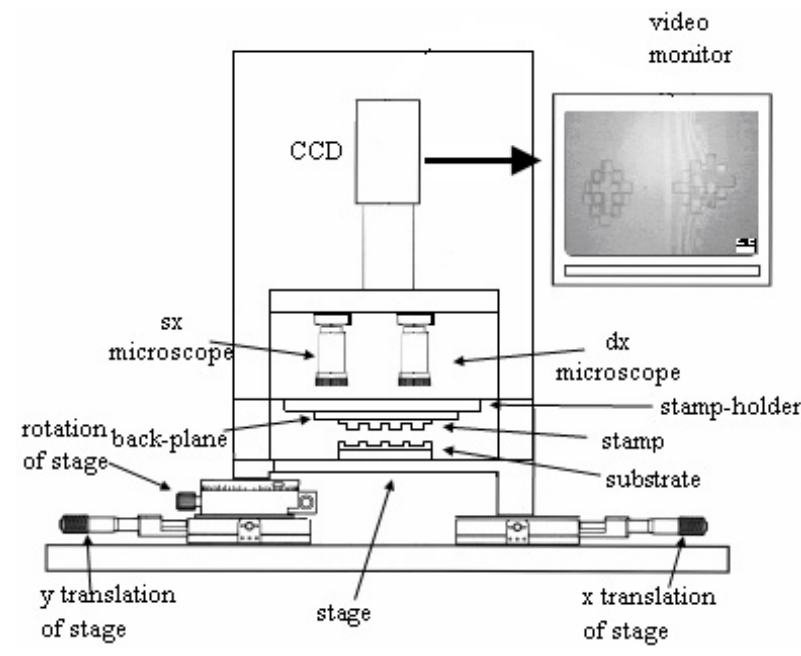

Figure 3. Layout of the alignment equipment for layer-by-layer lithography with soft stamps.

\subsection{Alignment}

The alignment process is carried out using state-of-the-art equipment (EVG 620) for layer-by-layer lithography with soft stamps, whose layout is illustrated in figure 3. A system of two optical microscopes connected to a charge coupled device camera and a monitor allows one to observe the top and the bottom layer during the alignment. The movement of the bottom layer stage relative to the top layer is controlled by micrometer screws, analogous to a mask aligner commonly used in semiconductor technology. In addition, a parallelism compensation is performed during the stamp-target approach. In the M-SL configuration the bottom layer is fixed on a stage by a vacuum chuck, whereas the top layer adheres on a quartz backplane, in its turn fixed to a stamp-holder by vacuum. The high transparency of PDMS, h-PDMS and TOPAS ${ }^{\circledR}$ in the visible range allows optical alignment through the stamp material by hand/eye coordination of the operator.

For alignment, we first bring the markers on both sides of the layers to approximately the same $y$ position (figure 4), using simultaneously the two observation optics. Then, the top and bottom layers are approached up to a relatively large distance (about $10^{3} \mu \mathrm{m}$ ), to avoid any possible contact, and aligned by the superposition of the markers. We iterate this operation many times for each couple of markers on the opposite sides, finally reaching a registration within 10$20 \mu \mathrm{m}$ for each couple of markers. Finally, we perform fine alignment directly on the two dots/holes central matrices, and progressively reduce the gap between the two M-SL films down to $10 \mu \mathrm{m}$.

\subsection{Measurements and data analysis}

Upon contact, the stamp and the backplane are released on the bottom layer, and after removing the backplane the samples are transported from the aligner to an optical microscope providing $100 \times$ magnification for visual inspection. The mutual contact between the two involved surfaces is enough to prevent relative movement during transportation. We realized more than 50 samples by M-SL using PDMS, h-PDMS and 
Table 1. Alignment data and statistical averages for the registration accuracy of $15 \mathrm{M}-\mathrm{SL}$ samples realized with PDMS, h-PDMS, and TOPAS $^{\circledR}$

\begin{tabular}{|c|c|c|c|c|c|c|c|c|c|}
\hline Material & $E(\mathrm{MPa})$ & $\langle\Delta x\rangle_{\mathrm{r}}(\mu \mathrm{m})$ & $\sigma_{x \mathrm{r}}(\mu \mathrm{m})$ & $\langle\Delta x\rangle_{\mathrm{c}}(\mu \mathrm{m})$ & $\sigma_{x \mathrm{c}}(\mu \mathrm{m})$ & $\langle\Delta y\rangle_{\mathrm{r}}(\mu \mathrm{m})$ & $\sigma_{y \mathrm{r}}(\mu \mathrm{m})$ & $\langle\Delta y\rangle_{\mathrm{c}}(\mu \mathrm{m})$ & $\sigma_{y \mathrm{c}}(\mu \mathrm{m})$ \\
\hline PDMS & 1.8 & 2.59 & 0.26 & 2.59 & 0.11 & 7.69 & 0.20 & 7.70 & 0.09 \\
\hline PDMS & 1.8 & 0.07 & 0.03 & 0.07 & 0.02 & 1.11 & 0.16 & 1.12 & 0.06 \\
\hline PDMS & 1.8 & 6.40 & 0.19 & 6.45 & 0.07 & 2.43 & 0.17 & 2.43 & 0.09 \\
\hline PDMS & 1.8 & 1.59 & 0.17 & 1.59 & 0.19 & 6.83 & 0.25 & 6.86 & 0.13 \\
\hline PDMS & 1.8 & 5.65 & 0.18 & 5.65 & 0.06 & 0.57 & 0.25 & 0.58 & 0.13 \\
\hline h-PDMS & 8.2 & 2.40 & 0.18 & 2.39 & 0.26 & 1.22 & 0.20 & 1.21 & 0.19 \\
\hline h-PDMS & 8.2 & 0.48 & 0.05 & 0.49 & 0.16 & 1.96 & 0.11 & 1.96 & 0.11 \\
\hline h-PDMS & 8.2 & 0.68 & 0.03 & 0.67 & 0.11 & 2.80 & 0.09 & 2.80 & 0.05 \\
\hline h-PDMS & 8.2 & 0.12 & 0.03 & 0.12 & 0.05 & 1.98 & 0.14 & 1.97 & 0.05 \\
\hline h-PDMS & 8.2 & 1.63 & 0.06 & 1.63 & 0.07 & 2.26 & 0.11 & 2.27 & 0.09 \\
\hline TOPAS & 2600 & 0.00 & 0.00 & 0.00 & 0.00 & 0.00 & 0.00 & 0.00 & 0.00 \\
\hline TOPAS & 2600 & 0.75 & 0.04 & 0.75 & 0.04 & 0.07 & 0.02 & 0.07 & 0.02 \\
\hline TOPAS & 2600 & 0.00 & 0.00 & 0.00 & 0.00 & 0.00 & 0.00 & 0.00 & 0.00 \\
\hline TOPAS & 2600 & 1.32 & 0.04 & 1.31 & 0.06 & 0.11 & 0.04 & 0.12 & 0.02 \\
\hline TOPAS & 2600 & 0.22 & 0.03 & 0.22 & 0.03 & 0.85 & 0.06 & 0.86 & 0.04 \\
\hline
\end{tabular}
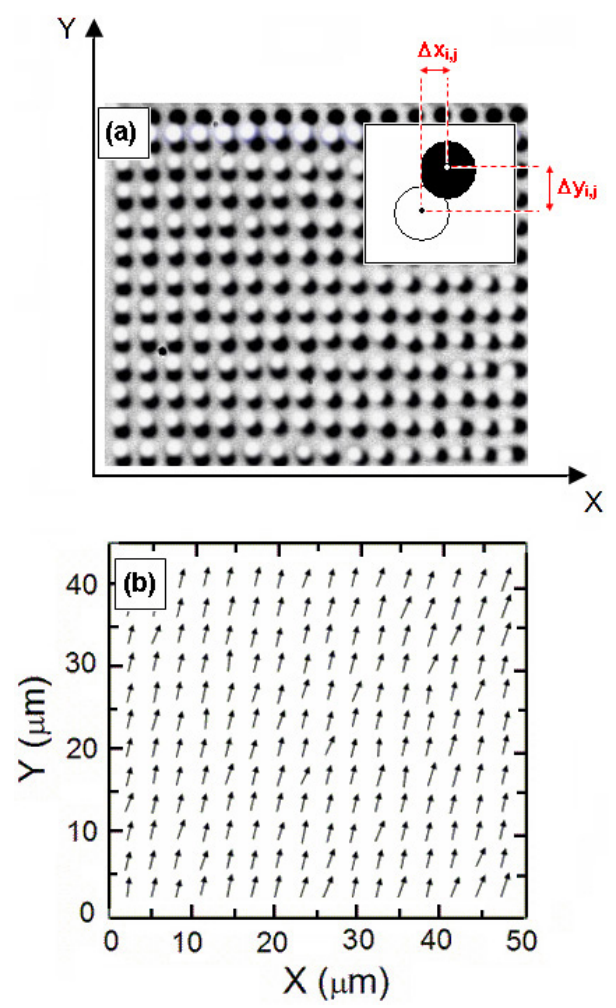

Figure 4. (a) False-colours optical micrograph of a M-SL sample: bottom polyurethane pitches are indicated in black, whereas the top layer features are displayed as white circles. Inset: scheme of the misalignment components $\left(\Delta x_{i, j}\right.$ and $\Delta y_{i, j}$ ) obtained for each pitch. (b) Misalignment map of vectors with components $\Delta x_{i, j}$ and $\Delta y_{i, j}$.

TOPAS $^{\circledR}$ as stamps for the top layer material. A detailed statistical analysis has been carried out on the optical images of the M-SL samples, determining the misalignment vectors between each pitch belonging to the bottom layer polyurethane matrix (white circles in figure 4(a)) and the corresponding hollow feature of the stamp (black circles). The misalignment vectors were determined along the lines connecting the centres of the bottom pitches and of the stamp hollow features, and assumed to lie in the $x y$ plane. Hence, a $16 \times 16$ displacements matrix is obtained for each M-SL sample (figure 4(b)), the generic misalignment vector having components $\Delta x_{i, j}$ and $\Delta y_{i, j}$, where the indexes $i$ and $j$ label the rows and the columns of the displacements matrix. For each row and each column we calculated the average value $\left\langle\Delta x_{i}\right\rangle\left(\left\langle\Delta y_{i}\right\rangle\right)$ of the $16 x(y)$ misalignment vector components on the $i$ row and the mean value $\left\langle\Delta x_{j}\right\rangle\left(\left\langle\Delta y_{j}\right\rangle\right)$ of the $16 x(y)$ components on the $j$ column. Moreover, for each sample we calculated the average values $\left\langle\Delta x_{\mathrm{R}}\right\rangle=\frac{1}{16} \sum_{i=1}^{16}\left\langle\Delta x_{i}\right\rangle\left(\left\langle\Delta y_{\mathrm{R}}\right\rangle=\frac{1}{16} \sum_{i=1}^{16}\left\langle\Delta y_{i}\right\rangle\right)$ of the misalignment components for rows and those for columns $\left(\left\langle\Delta x_{\mathrm{C}}\right\rangle=\frac{1}{16} \sum_{j=1}^{16}\left\langle\Delta x_{j}\right\rangle\right.$ and $\left.\left\langle\Delta y_{\mathrm{C}}\right\rangle=\frac{1}{16} \sum_{j=1}^{16}\left\langle\Delta y_{j}\right\rangle\right)$, and the corresponding standard deviations, $\sigma_{x, \mathrm{R}}, \sigma_{y, \mathrm{R}}, \sigma_{x, \mathrm{C}}$, and $\sigma_{y, \mathrm{C}}$.

\section{Results and discussion}

Similarly to nanoimprinting, M-SL alignment steps have to be continuously carried out while bringing the stamp in contact with the target film. Methods employing registration markers external to the patterned area are suitable for their simplicity and because the achieved optical contrast is not reduced by the soft lithography process, such as in the case of channels progressively filled by polymer solution in micromoulding in capillaries [1].

The obtained results for 15 prototype M-SL samples are summarized in table 1. By employing Sylgard 184 PDMS $(E=1.8 \mathrm{MPa})$ [31], h-PDMS $(E=8.2 \mathrm{MPa})$ [31] and $\operatorname{TOPAS}^{\circledR}(E=2600 \mathrm{MPa})$ [20], we found an average value of the misalignment vector modulus over all the pitch/hole couples, of $(4.96 \pm 0.02) \mu \mathrm{m},(2.29 \pm 0.01) \mu \mathrm{m}$, and $(0.50 \pm$ $0.01) \mu \mathrm{m}$, respectively. In particular, in some samples realized with TOPAS ${ }^{\circledR}$ we could obtain a complete alignment, resulting in the penetration of the bottom polyurethane dots into the hollow features of the top layer. Therefore, in these cases (occurring in $40 \%$ of the TOPAS ${ }^{\circledR}$ M-SL specimens) we infer that a registration accuracy within the range of tens of nanometres can be achieved, since the bottom dots exhibit sides $10 \mathrm{~nm}$ smaller than those of the dots on the top layer. The overall registration accuracy achievable with rigid replicas over our operation area $\left(50 \times 50 \mu \mathrm{m}^{2}\right)$ is comparable with the optical accuracy provided by the employed alignment equipment. 
This registration method could be applied to real soft lithography processes by properly treating the M-SL layers, depending on the particular chosen technology. For instance, in order to print proteins the patterned surface of the top layer should be first inked by a biomolecule solution, then dried, as in the usual $\mu \mathrm{CP}$ procedure, and finally positioned in the aligner for registration. The implemented alignment process requires about $5 \mathrm{~min}$ after positioning the layers in the aligners, which is fully compatible with the typical times used for printing biomolecules, and, since it is carried out by light of wavelengths above $670 \mathrm{~nm}$, it retains the protein functionality. Overall, we estimate for a complete M-SL process (inking, drying, aligning and printing) a throughput of two samples printed per hour

In our experiments, by observing the misalignment of the reference markers at the end of the registration iterations, we found an alignment accuracy in the range of 10-20 $\mu \mathrm{m}$ between the two M-SL layers for the overall $3.5 \times 3.5 \mathrm{~cm}^{2}$ sample area. Implementing large-area alignment iterative procedures would be facilitated by stereomicroscopes allowing one to visualize simultaneously many markers with high optical magnification $(\geqslant 10 \times$ on areas of a few square centimetres). This strategy could lead to finding similar values (below $1 \mu \mathrm{m}$ ) for the alignment accuracy achieved locally (i.e. on sample areas in the range of $10^{3} \mu \mathrm{m}^{2}$ ) and globally (sample areas of a few square centimetres) by rigid replicas. Large focus distance (of the order of a few centimetres) would also benefit alignment of elastomeric elements for microfluidic applications, often requiring thicker polymeric layers for ensuring mechanical stability to the assembled devices and easier connections with external capillaries.

In fact, while in multilevel nanoimprinting lithography the main sources of misalignment are the non-perfect parallelism between the stamp and the target film, non-uniform pressures and temperatures, different thermal expansions in the resist and in the stamp, and possible wafer bending during imprinting [28], the registration achievable in soft lithographies, mostly working at ambient temperature and pressure, is crucially affected by the elastic properties of the polymeric replicas. Soft elastomeric materials, such as organic polymers with Young's modulus lower than 9.0 MPa [33], are more affected by pattern deformations and collapse of the structures when a patterned stamp goes into contact with a rough substrate. Elastomers can also undergo local sagging of the top layer when one side region of the stamp is significantly thicker (in our samples we estimate this variation around 10\%) as reported by Hu et al [14]. In particular, elastomeric elements spontaneously tend to adhere to the target surface and to prime a propagation front of conformal contact. Once the contact occurs in a certain location, it rapidly progresses along the target surface, driven by the difference between the total stamp and polyurethane surface energies $\left(\gamma_{\mathrm{ELAST}}+\gamma_{\mathrm{NOA}}\right)$ and the interfacial energy at the contact surface. This may determine a non-uniform stamp-target gap during approaching and concomitant distortions of the top layer because of the induced strain, resulting in loss of parallelism and misalignments in the M-SL.

Our analysis on the M-SL performance provides insight into the distortion issue, since comparing the alignment accuracy and its standard deviation obtained for many points and for rows and columns in our squared geometry is a measurement of the registration disuniformity and of the deformations occurring throughout the polymeric patterns. For instance, the standard deviations $\sigma_{x, \mathrm{R}}, \sigma_{y, \mathrm{R}}, \sigma_{x, \mathrm{C}}$ and $\sigma_{y, \mathrm{C}}$ give a measurement of the width of the distribution of the misalignment vectors along the directions, $x$ and $y$, for the rows and the columns of each sample. We note that MSL samples with PDMS top layer do not exhibit correlation between the values of the standard deviations relative to rows and columns (table 1), namely $\sigma_{x, \mathrm{R}}\left(\sigma_{y, \mathrm{R}}\right)$ may differ significantly from $\sigma_{x, \mathrm{C}}\left(\sigma_{y, \mathrm{C}}\right)$ for each sample. Considering all the realized PDMS-based samples, we found a $50 \%$ probability that the standard deviations of the components of the misalignment vector change by more than a factor of two from rows to columns, which is an indication that remarkable distortions occur in the top layer pattern. The row-column misalignment correlation increases for the samples with hPDMS top layer, for which the above mentioned probability is around $30 \%$. Finally, the M-SL samples with TOPAS ${ }^{\circledR}$ top layer exhibit only a $10 \%$ probability of a change by more than a factor of two in the standard deviations of the components of the misalignment vectors for rows and columns, which is attributable to purely statistical variations, similarly to results reported about alignment processes for nanoimprinting lithography with silicon templates [27]. These findings confirm the remarkably lower presence of distortions in patterns defined in harder polymers, and its relationship with a better registration accuracy achievable in M-SL.

\section{Conclusion}

In summary, we investigated the registration accuracy achievable for M-SL. We obtained for the average misalignments over areas of $50 \times 50 \mu \mathrm{m}^{2}$ values decreasing from $(4.96 \pm$ $0.02) \mu \mathrm{m}$ to $(0.50 \pm 0.01) \mu \mathrm{m}$ upon increasing the Young's modulus of the stamp materials from 1.8 to $2600 \mathrm{MPa}$. This clearly identifies the replica distortions as main limiting factors for the registration accuracy. Multilevel soft lithographies will have to find a compromise between the ease of processing and conformability of the stamps, and the pattern stability favouring registration, depending on the particular application.

\section{Acknowledgments}

This work was partially funded by the Istituto Italiano di Tecnologia (IIT). We also thank the support of the Italian Minister of Education, University and Research through the FIRB project 'Laboratorio nazionale sulle nanotecnologie per la genomica e postgenomica' (NG-Lab).

\section{References}

[1] Xia Y and Whitesides G M 1998 Angew. Chem. Int. Edn 37550

[2] Chou S Y, Krauss P R and Renstrom P J 1996 Science 27285

[3] Austin M D, Ge H, Wu W, Li M, Yu Z, Wasserman D, Lyon S A and Chou S Y 2004 Appl. Phys. Lett. 845299

[4] Li M, Wang J, Zhuang L and Chou S Y 2000 Appl. Phys. Lett. 76673

[5] Zhang W and Chou S Y 2003 Appl. Phys. Lett. 831632

[6] http://www.itrs.net/Links/2003ITRS/Litho2003.pdf 
[7] Keil M, Beck M, Frennesson G, Theander E, Bolmsjö E, Montelius L and Heidari B 2004 J. Vac. Sci. Technol. B 223283

[8] Rowland H D, King W P, Sun A C and Schunk P R 2005 J. Vac. Sci. Technol. B 232958

[9] Xia Y, McClelland J J, Gupta R, Qin D, Zhao X M, Sohn L L, Celotta R J and Whitesides G M 1997 Adv. Mater. 9147

[10] Kumar A and Whitesides G M 1993 Appl. Phys. Lett. 632002

[11] Zaumseil J, Meitl M A, Hsu J W, Acharya B, Baldwin K W, Loo Y L and Rogers J A 2003 Nano Lett. 31223

[12] Chang-Yen D A, Eich R K and Gale B K 2005 J. Lightwave Technol. 232088

[13] Kim C, Cao Y, Soboyevo W O and Forrest S R 2005 J. Appl. Phys. 97113512

[14] Hu J, Beck R G, Deng T, Westervelt R M, Maranowski K D, Gossard A C and Whitesides G M 1997 Appl. Phys. Lett. 712020

[15] Whitesides G M, Ostuni E, Takayama S, Jiang X and Ingber D E 2001 Annu. Rev. Biomed. Eng. 3335

[16] Love J C, Estroff L A, Kriebel J K, Nuzzo R G and Whitesides G M 2005 Chem. Rev. 1051103

[17] Chen C S, Mrksich M, Huang S, Whitesides G M and Ingber D E 1997 Science 2761425

[18] Gates B D and Whitesides G M 2003 J. Am. Chem. Soc. 12514986
[19] Mele E, Di Benedetto F, Persano L, Pisignano D and Cingolani R 2005 Nanotechnology 16391

[20] Nielsen T, Nilsson D, Bundgaard F, Shi P, Szabo P, Geschke O and Kristensen A 2004 J. Vac. Sci. Technol. B 221170

[21] Plachetka U, Bender M, Fuchs A, Vratzov B, Glinsner T, Lindner F and Kurz H 2004 Microelectron. Eng. 73/74 167

[22] Renault J P, Bernard A, Juncker D, Michel B, Bosshard H R and Delamarche E 2002 Angew. Chem. 1142426

[23] Unger M A, Chou H P, Thorsen T, Scherer A and Quake S R 2000 Science $\mathbf{2 8 8} 113$

[24] Hsu T R 2002 MEMS and Microsystems: Design and Manufacturing (Boston, MA: McGraw-Hill)

[25] Toepke M W and Kenis P J A 2005 J. Am. Chem. Soc. 1277674

[26] Ke C J, Yi X J, Lai J J and Chen S H 2005 Int. J. Infrared Millim. Waves 26133

[27] Zhang W and Chou S Y 2001 Appl. Phys. Lett. 79845

[28] Li N, Wu W and Chou S Y 2006 Nano Lett. 62626

[29] Lee J H, Kim C H, Kim Y S, Ho K M, Constant K, Leung W and Oh C H 2005 Appl. Phys. Lett. 86204101

[30] Rogers J A, Paul K E and Whitesides G M 1998 J. Vac. Sci. Technol. B 1688

[31] Choi K M and Rogers J A 2003 J. Am. Chem. Soc. 1254060

[32] http://www.norlandprod.com

[33] Bietsch A and Michel B 2000 J. Appl. Phys. 884310 